\title{
THE EFFECT OF VOLUNTARY AND POST-HYPNOTIC ATTENTION ON THE RECALL OF DIGITS ${ }^{1}$
}

\author{
GOSAKU NARUSE ${ }^{2}$ \\ Kyushu University
}

\begin{abstract}
Using as Ss 25 secondary school children selected for their yielding of suggested post-hypnotic amnesia, recall of digits was tested under 5 conditions: I. Control condition, 9 homogeneous digits : II. Control condition, heterogeneous material, seventh position with letter of the alphabet substituted for a digit : III. Active voluntary attention to seventh digit: IV. Passive voluntary attention (posthypnotic) to seventh digit: V. Passive voluntary inhibition (post-hypnotic) of seventh digit.

The von Restorff effect (accentuation of seventh item) was shown in the control condition with the letter as a heterogeneous item, and also demonstrated under condition of both active and passive attention to the digit in the seventh position. The passive voluntary condition differed, however, from the active voluntary condition.
\end{abstract}

Attention has sometimes been divided into non-voluntary orientation toward a stimulus and voluntary orientation, the former occasionally referring more to senseorgan adjustment, the latter to adjustments of the whole organism. Voluntary attention may be further differentiated into active and passive types, depending upon the amount of effort and deliberate goaldirection involved (Harano, Ogawa, \& Naruse, 1965).

The "von Restorf effect" in memory illustrates non-voluntary orientation, in that the subject is attracted to the isolated part of the configuration. To illustrate active voluntary attention, a subject may be told to direct attention to some particular part of a series of digits containing no structural "isolation." To obtain the passive type of voluntary attention, a post-hypnotic

1 This research was supported by grant in Aid for Scientific Research 1959-1960 from the Ministry of Education.

2 The author is indebted to Dr. E. R. Hilgard, Stanford University, for critical reading this manuscript and making invaluable suggestions relative to English phraseology, and also to Dr. S. Iwahara, Tokyo University of Education, for helpful advices concerning statistical examination. suggestion can be given to the same end as the active instruction. This paper reports a comparative study of these three types of attention (non-voluntary, active voluntary, and passive voluntary) as they affect the recall of digits.

\section{MethoD}

The material for the study of memory is of two kinds: homogeneous and heterogeneous. The homogeneous material consists of the 9 digits, 1-9, arranged in random order. The heterogeneous material is the same, except that in the seventh position a letter of the alphabet is substituted for a digit.

In addition to the active and passive voluntary conditions to be compared, there were two control conditions for non-voluntary effects, Condition I, with the homogeneous material, and Condition II with the heterogeneous material. The 9 items in a horizontal line were presented at once visually for two seconds. After presentation, the $S$ was asked to write the digits that he remembered. Conditions I and II were run in immediate succession.

The remaining conditions, III, IV, and 
$\mathrm{V}$ were as follows:

III. Active voluntary attention. $S$ 's attention was directed specifically to the seventh figure. He was asked to memorize it particularly well, and after recalling the whole span of digits to write the figure in the appointed space. Otherwise the instructions were the same as for conditions I and II.

IV. Passive voluntary attention: post-hypnotic. The $S$ received the same instruction as in condition III, but as a post-hypnotic suggestion under hypnosis and with amnesia for the suggestion. Hence before the digits were presented, the following procedures had been followed: (i) induction of deep hypnosis, tested by $S$ 's inability to remember his age when such amnesia was suggested, (ii) the $S$, while hypnotized, was given the instructions of Condition III to be carried out post-hypnotically with amnesia for the fact that such instructions had been given, (iii) the subject was dehypnotized and questioned to confirm the fact of post-hypnotic amnesia, and (iv) the memory material was given, now with the neutral instructions of Condition I.

V. Passive voluntary inhibited attention: post-hypnotic. This condition was like Condition IV except that, instead of emphasizing the retention of the seventh item, the $S$ received the post-hypnotic suggestion that he could not memorize the seventh figure and could not recall it.

The $25 \mathrm{Ss}$ were selected from a large number aged 13-15 from class-rooms of the secondary school. Only those were selected who had proved amnesic for their age after this amnesia was suggested. The conditions were presented in the order I to $\mathrm{V}$ for all $S \mathrm{~s}$. Conditions I and II were given in immediate succession, followed, after completing the instructions, by condition III. Then 30 minutes elapsed before Condition IV, and 60 minutes between Condition IV and V. Each condition was composed of three trials of memorization, that is, $S$ s were asked to respond to three different materials.
TABLE 1

Results of $F$-analysis of 5 conditions, 9 positions of digit, and $25 \mathrm{Ss}$

\begin{tabular}{lrrrc}
\hline \multicolumn{1}{c}{ Variables } & $S S$ & $d f$ & $M S$ & $F$ \\
\hline Condition (A) & 4.38 & 4 & 1.095 & 0.890 \\
Position (B) & 475.45 & 8 & 59.431 & $56.067^{* *}$ \\
Subject (C) & 181.02 & 24 & 7.543 & $69.265^{* *}$ \\
$\mathrm{~A} \times \mathrm{B}$ & 87.56 & 32 & 2.643 & $24.248^{* *}$ \\
$\mathrm{~A} \times \mathrm{C}$ & 117.84 & 96 & 1.23 & $11.28^{* *}$ \\
$\mathrm{~B} \times \mathrm{C}$ & 204.04 & 192 & 1.06 & $9.72^{* *}$ \\
$\mathrm{~A} \times \mathrm{B} \times \mathrm{C}$ & 83.62 & 768 & 0.109 & \\
\hline Total & 1150.91 & 1124 & & \\
$* *$ shows $p<.01$ & & &
\end{tabular}

\section{RESULTS}

Analysis of variance. By assigning 1 point for each digit correctly reported in its correct position, we would give 3 points to the subjects for each digital position when they responded correctly always on three trials in each condition. Here, it is possible to arrange scores for an analysis of variance.

The results of such an analysis for 5 conditions (A), 9 positions of the digit (B), and $25 \mathrm{Ss}(\mathrm{C})$, taking into account the correlations that are involved because the same $S$ s yielded the data for all conditions and for all positions, are presented in Table 1 .

Because the interaction $\mathrm{A} \times \mathrm{B}$ (condition $\times$ position) are significant, we are free to use $T$-test to locate the places where the differences arise.

Differences of initial position by condition. As expected, the first position has the highest recall under all conditions. For 4 of the conditions (I, II, IV, and V) there are no significant differences. However, condition III, in which there was voluntary active interaction to attend to the seventh digit, responses to the first digit were depressed by a significant amount ( $T$-test, $p<.01$ ) below the score for each of the other conditions.

Control comparisons at position 7. The only differences in the construction of the lists for conditions I and II was the appearance 
of a letter at position 7 in condition II. This resulted in the von Restorff effect of heightened response to the isolated letter, producing an overall difference between the two conditions (F-test, $p<.01)$. Fig. 1 shows the mean recall at each position for condition I and II, with the striking hump at position 7 for condition II.

Effect of active voluntary attention. The appropriate comparison between the active voluntary attention to the seventh item and the control condition with all digits is that between condition III and condition I, shown in Fig. 2. The curves differ significantly $(F$-test, $p<0.01)$. The means are significantly different at position 7 ( $T$-test, $p<0.01)$. Apparently the accentuation at position 7 carries with it an enhancement of recall for position 8 and 9, with a corresponding decrement in responses at positions 1 to 4.

Effect of passive voluntary attention (posthypnotic). While the instructions given posthypnotically for condition IV were essentially those of condition III, it turns

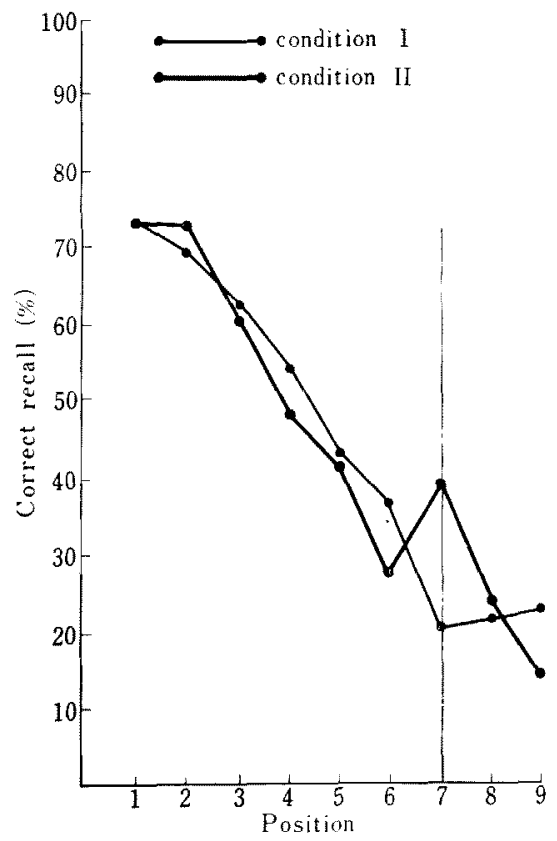

Frg. 1. Curves of mean percentage of correct recall at each position in condition $I$ and $I I$. out that the effect of these passive instructions was to yield a result similar not to condition III but to the nonvoluntary accentuation of condition II, in which the seventh position was isolated by a letter placed among the digits rather than by instructions (Fig. 3). Thus conditions IV and III differ significantly ( $F$-test, $p<0.01$ ), while conditions IV and II do not differ significantly.

Effect of passive voluntary inhibition (posthypnotic). The corresponding comparisons are given in Fig. 4 for the instruction to fail on position 7 (condition V), compared with conditions I and IV. It might have been conjectured that this instruction would have reduced the responsiveness at the seventh position below that of control condition I, but this did not happen. What did happen was that there were no significant overall differences between conditions I, IV, and V. Apparently the attention to the seventh item, even though it followed the suggestion that the item would not be recalled, did something to enhance atten-

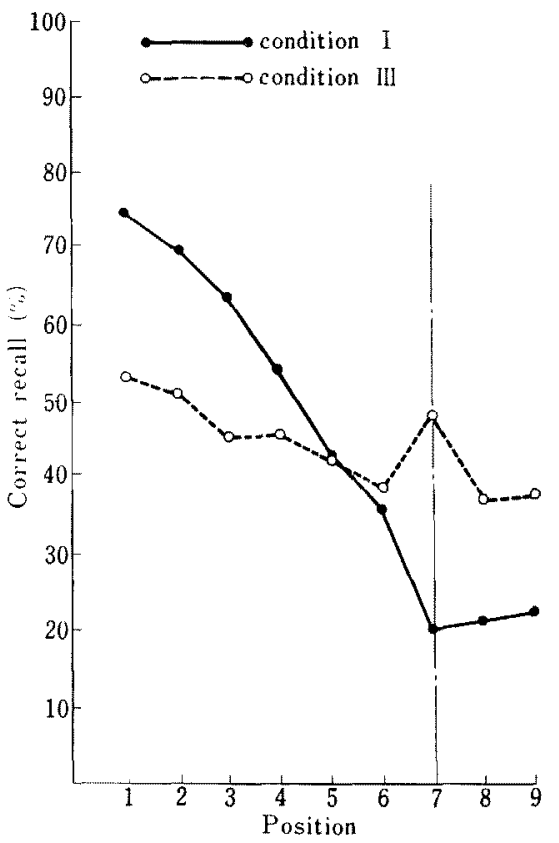

FIG. 2. Curves of mean percent of correct recall of condition I and III. 
tiveness to items in its neighborhood; even though there is no peak at the seventh item, items 6,7 , and 8 lie above the corresponding items for condition IV. These results call for further discussion.

\section{Discussion}

Heretofore the effect of an isolated part on memory (the von Restorff effect) has been discussed from the point of view of the stimulus configuration (e.g., Obonai, 1961). The present study shows, however, that a similar effect can be produced within a set of homogeneous digits if the $S$ 's attention is directed to a corresponding digit by post-hypnotic suggestion, as in condition IV. Thus the distribution of attention affected either by the structure of the stimulus or by the $S$ 's striving may produce similar effects on memory.

The difference between the active voluntary direction of attention in the waking state and the passive voluntary attention produced by the same instructions operat-

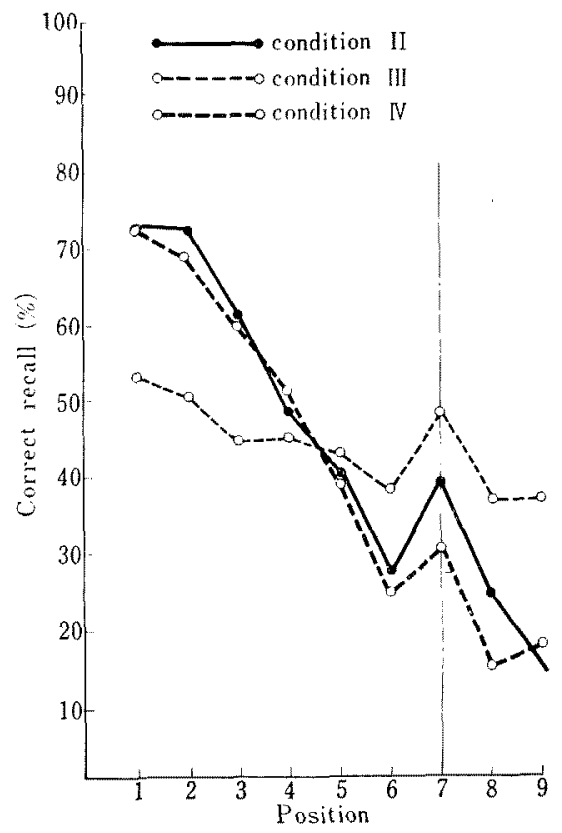

FIG. 3. Gurves of mean percent of correct recall of condition II, III and IV. ing as post-hypnotic suggestions (with amnesia for the suggestions) was shown in the comparison between the results of condition III and condition IV. Thus the hypothesized distinction between an active or conscious type of voluntary attention, and a passive or subconscious type is borne out.

These results may cause some researchers to reconsider their motivational theory of hypnosis, in view of their neglect of the differences in the striving by the normal waking $S$ and by the hypnotic or posthypnotic $S$ (Barber and Calverley, 1962). Barber's notion of task motivation must take into account a difference in effect between the usual type of task motivation and the type found in hypnosis or as a result of post-hypnotic suggestion.

The failure of the inhibiting instructions of condition $V$ to produce a fall in score at the seventh position calls for some comments. It appears that in the post-hypnotic state the calling attention to the seventh position, even though accompanied by the

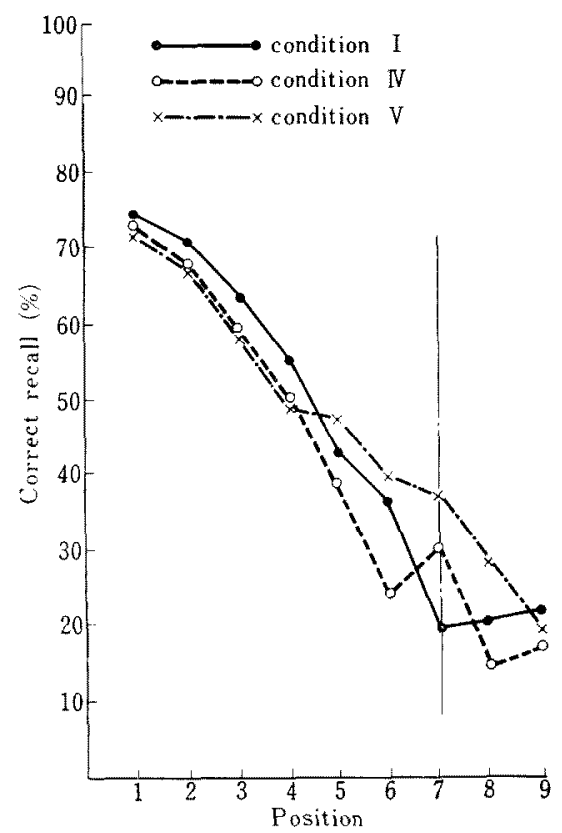

Frg, 4. Curves of mean percent of correct recall of condition I, IV, and V. 
suggestion that recall would be difficult, had a positive effect, just as a warning "Wet Paint" sometimes invites sticking one's finger in it. This positive effect was more diffuse than when the positive suggestion was given directly (as in condition IV), and spread over the adjoining positions. In view of the amnesia, it may be that the subconscious instruction operated in a confused manner, as though it meant "there's something important about the seventh position." A full understanding of what was happening awaits further experimentation.

The experimental design is somewhat faulty in that the same $25 \mathrm{~S}$ s served in all conditions and in the same order. While some order effects cannot therefore be analyzed, the results are striking enough by condition that it does not appear that they are created by any progressive practice effect. It is proposed, however, to carry out additional experiments in which there will be different groups under each of the five conditions in order to eliminate any possible order effects.

\section{REFERENCES}

Barber, T.X., \& Calverley, D. S. 1962 " Hypnotic behavior" as a function of task motivation. f. abnorm. soc. Psychol., 66, 589-597.

Harano, K., Ogawa, K., \& Naruse, G. 1965 A study of plethysmography and autogenic exercise. In W. Luthe (Ed.), Autogenes Training: Correlations psychosomaticae. Stuttgart: Georg Thieme, Pp. 55-58.

NARUSE, G, 1965 A genetic consideration of hypnotic hallucination. Jap. F. educ. soc. Psychol., 5, 15-19.

Oвons1, T. 1961 Kioku to Shiko (Memory \& Thinking). Tokyo: Nakayama.

(Received May 6, 1966) 